\title{
Coupling Of Alpha- And t-cluster Structures In Excited Deformed States Of $35 \mathrm{Cl}$
}

Yasutaka Taniguchi*

Nihon Institute of Medical Science

E-mail: yasutakadnims.ac.jp

Clustering and deformation are important for dynamics of nuclear structure, and cluster structures couple to deformed states. However, its mechanism is unclear. Cluster structures that couple to each deformed state and the mechanism are investigated in ${ }^{35} \mathrm{Cl}$. The antisymmetrized molecular dynamics (AMD) and the generator coordinate method (GCM) are used. An AMD function is a Slater determinant of Gaussian wave packets. By energy variational calculations with constraints for deformation and clustering, wave functions that have deformed and $\alpha-{ }^{31} \mathrm{P}$ and $t-{ }^{32} \mathrm{~S}$ cluster structures are obtained. Adopting those wave functions as GCM basis, wave functions of ground and excited states are calculated. Various cluster deformed bands are obtained and predicted. The $K^{\pi}=\frac{1}{2}^{-}$deformed bands contains large amount of $\alpha-{ }^{31} \mathrm{P}$ and $t-{ }^{32} \mathrm{~S}$ cluster structure components. Particle-hole configurations of both cluster structures are same as dominant components of the $K^{\pi}=\frac{1}{2}^{-}$bands in small intercluster distance. Particle-hole configurations of cluster structure in small intercluster distance are important for coupling of cluster structure to deformed states.

The 26th International Nuclear Physics Conference

11-16 September, 2016

Adelaide, Australia

* Speaker. 


\section{Introduction}

Nuclear structure changes drastically under low-excitation energies. A goal of nuclear physics is to understand structure changes simply and microscopically. Clustering and deformation play important roles for nuclear structure. Cluster structures couple to deformed states. In order to understand structure of deformed states, cluster structure coupling to deformed states are important. Two rules have been proposed to predict cluster structures coupling to deformed states. One is the threshold rule in which cluster structures are developed in excited states whose excitation energies are similar to threshold energies of cluster decay. Another one is particle-hole configurations. When nucleons are excited to higher shell and those nucleons correlate strongly, they are expected to form a cluster.

In ${ }^{35} \mathrm{Cl}$, a negative-parity rotational bands have been observed from $J^{\pi}=\frac{15}{2}^{-}(8.31 \mathrm{MeV})$ to $\frac{27}{2}^{(-)}(16.30 \mathrm{MeV})$ states by a $\gamma$-spectroscopy experiment[四]. In-band $B(\mathrm{E} 2)$ values are deduced, and the values are around $30 \mathrm{~W} . \mathrm{u}$, which shows the band form largely deformed structure. In the experimental paper, $\alpha{ }^{-32} \mathrm{~S}$ cluster structure with one proton hole of the band is discussed with mentioning threshold energies of $\alpha+{ }^{31} \mathrm{P}$ and $t+{ }^{32} \mathrm{~S}$ channels, which are $6.99 \mathrm{MeV}$ and $17.94 \mathrm{MeV}$, respectively. However, details of cluster coupling of the band are not discussed, and theoretical work of cluster structure are insufficient in ${ }^{35} \mathrm{Cl}$.

This paper aims to clarify $\alpha{ }^{-}{ }^{31} \mathrm{P}$ and $t{ }^{32} \mathrm{~S}$ cluster coupling to deformed states in ${ }^{35} \mathrm{Cl}$. By the antisymmetrized molecular dynamics (AMD) and the generator coordinate method (GCM), various structures including superdeformed (SD) states are obtained. By analyzing of wave functions of the deformed states and the cluster structures, it is found that particle-hole configuration are important for coupling of cluster structure to deformed states.

\section{Framework}

In this section, the framework of the study is explained briefly. The details of the framework are provided in Refs. [园, 目, 团].

The wave functions in low-lying states are obtained using parity projection and angular momentum projection (AMP) and the GCM with deformed-basis AMD wave functions. A deformedbasis AMD wave function $|\Phi\rangle$ is a Slater determinant of Gaussian wave packets that can deform triaxially such that

$$
\begin{gathered}
|\Phi\rangle=\hat{\mathscr{A}}\left|\varphi_{1}, \varphi_{2}, \cdots, \varphi_{A}\right\rangle, \\
\left|\varphi_{i}\right\rangle=\left|\phi_{i}\right\rangle \otimes\left|\chi_{i}\right\rangle \otimes\left|\tau_{i}\right\rangle, \\
\left\langle\mathbf{r} \mid \phi_{i}\right\rangle=\left(\frac{\operatorname{det} \mathrm{M}}{\pi^{3}}\right)^{1 / 4} \exp \left[-\frac{1}{2}\left(\mathbf{r}-\mathbf{Z}_{i}\right) \cdot \mathrm{M}\left(\mathbf{r}-\mathbf{Z}_{i}\right)\right], \\
\left|\chi_{i}\right\rangle=\chi_{i}^{\uparrow}|\uparrow\rangle+\chi_{i}^{\downarrow}|\downarrow\rangle, \\
\left|\tau_{i}\right\rangle=|\pi\rangle \text { or }|v\rangle,
\end{gathered}
$$

where $\hat{A}$ denotes the antisymmetrization operator, and $\left|\varphi_{i}\right\rangle$ denotes a single-particle wave function. Further, $\left|\phi_{i}\right\rangle,\left|\chi_{i}\right\rangle$, and $\left|\tau_{i}\right\rangle$ denote the spatial, spin, and isospin components, respectively, of each single-particle wave function $\left|\varphi_{i}\right\rangle$. The real $3 \times 3$ matrix $\mathrm{M}$ denotes the width of the Gaussian single-particle wave functions that can deform triaxially and is common to all nucleons. $\mathbf{Z}_{i}=$ 
$\left(Z_{i x}, Z_{i y}, Z_{i z}\right)$ are complex parameters denoting the centroid of each single-particle wave function in phase space. The complex parameters $\chi_{i}^{\uparrow}$ and $\chi_{i}^{\downarrow}$ denote the spin directions. Axial symmetry is not assumed.

The basis wave functions of the GCM are obtained by energy variation with a constraint potential $V_{\text {cnst }}$ after projection onto eigenstates of parity,

$$
\begin{gathered}
\delta\left(\frac{\left\langle\Phi^{\pi}|\hat{H}| \Phi^{\pi}\right\rangle}{\left\langle\Phi^{\pi} \mid \Phi^{\pi}\right\rangle}+V_{\text {cnst }}\right)=0, \\
\left|\Phi^{\pi}\right\rangle=\frac{1+\pi \hat{P}_{r}}{2}|\Phi\rangle,
\end{gathered}
$$

where $\hat{H}$ and $\hat{P}_{r}$ denote the Hamiltonian and parity operator, respectively. The variational parameters are $\mathrm{K}, \mathbf{Z}_{i}$, and $\chi_{i}^{\uparrow, \downarrow}(i=1, \ldots, A)$. The isospin component of each single-particle wave function is fixed as a proton $(\pi)$ or a neutron $(v)$. The Gogny D1S force is used as the effective interaction.

To obtain the various wave functions, the constraint potential $V_{\text {cnst }}$ are added. In this work, two kinds of constraint potentials, matter quadrupole deformation parameter $\beta$ and intercluster distance [团], are used. For intercluster distance constraint, distance $d_{\alpha^{-31} \mathrm{P}}$ between $\alpha$ and ${ }^{31} \mathrm{P}$ and $d_{t-32} \mathrm{~S}$ between $t$ and ${ }^{32} \mathrm{~S}$ are constrained.

The optimized wave functions are superposed after parity projection and AMP by employing the quadrupole deformation parameter $\beta$ and intercluster distance constraints,

$$
\left|\Phi_{M}^{J \pi}\right\rangle=\sum_{i} \hat{P}_{M K_{i}}^{J \pi}\left|\Phi^{c_{i}}\left(q_{i}\right)\right\rangle f_{i}
$$

where $\hat{P}_{M K}^{J^{\pi}}$ is the parity and total angular momentum projection operator, and $\left|\Phi^{c_{i}}\left(q_{i}\right)\right\rangle$ is a basis wave function. $c_{i}$ is a kind of constraint potential, which are quadrupole deformation parameter $\beta$ and intercluster distance $d_{\alpha^{-31} \mathrm{P}}$ and $d_{t^{-32} \mathrm{~S}}$. Those parameters are constrained to $q_{i}$ by adding constraint potentials with parabola form. The integrals over the three Euler angles in the total angular momentum projection operator $\hat{P}_{M K}^{J}$ are evaluated by numerical integration. The numbers of sampling points $\left(N_{\alpha}, N_{\beta}, N_{\gamma}\right)$ in the numerical integration for $\alpha, \beta$, and $\gamma$ are $\left(N_{\alpha}, N_{\beta}, N_{\gamma}\right)=$ $(29,25,29)$ and $(26,25,26)$ for positive- and negative-parity, respectively. Here the body-fixed $z$ axis is determined by minimizing variance of $\hat{J}_{z}$, which is $z$ component of angular momentum. The coefficients $f_{i}$ are determined by the Hill-Wheeler equation,

$$
\delta\left(\left\langle\Phi_{M}^{J \pi}|\hat{H}| \Phi_{M}^{J \pi}\right\rangle-\varepsilon\left\langle\Phi_{M}^{J \pi} \mid \Phi_{M}^{J \pi}\right\rangle\right)=0 .
$$

Then we obtain the energy spectra and the corresponding wave functions, which are expressed by the superposition of the optimum wave functions.

\section{Results}

Figures $\square$ and $\square$ show energy curves as functions of quadrupole deformation parameter $\beta$ obtained by energy variational calculation with parity projection. In both parity, various structures are obtained, and particle-hole configurations are labeled in the figures. $(p f)_{\tau}^{n}(\tau=\pi$ or $v)$ denotes that $n$ proton(s) $(\pi)$ or neutrons(s) is/are excited from $s d$-shell to $p f$-shell.

In positive-parity states (Fig. $\mathbb{W}$ ), the lowest energy state exist at $\beta \simeq 0.2$ in which a proton nor a neutron excite to $p f$-shell. In deformed region, wave functions that have $(p f)_{\pi}^{1}(p f)_{v}^{1}$ and 


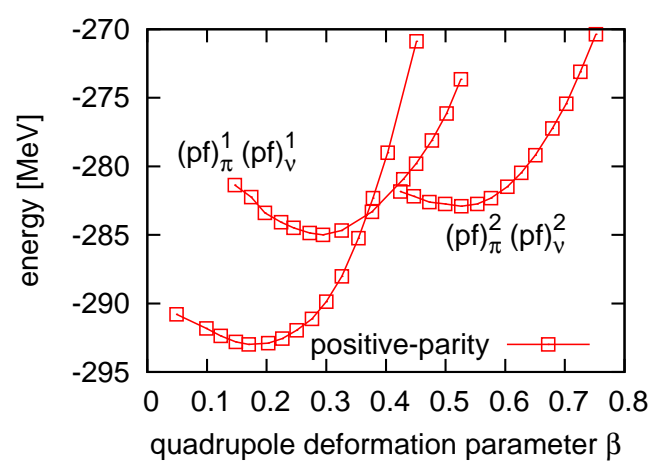

Figure 1: Energy curves as functions of quadrupole deformation parameter $\beta$ obtained by energy variational calculation with parity projection for positive-parity. $(p f)_{\tau}^{n}(\tau=\pi$ or $v)$ shows particle-hole configurations.

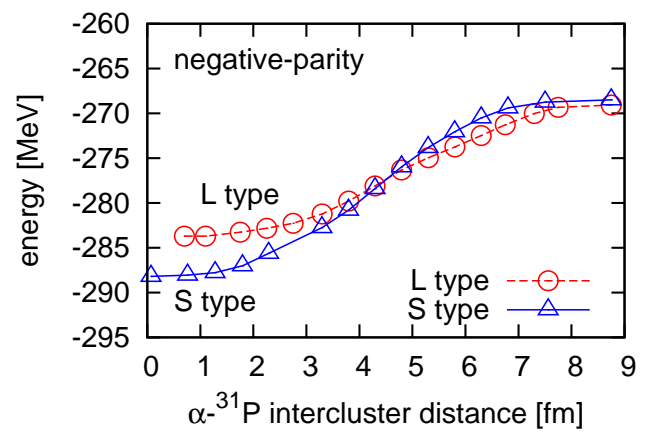

Figure 3: Energies of $\alpha{ }^{31} \mathrm{P}$ cluster structure as functions of intercluster distance for negativeparity states. Solid and dotted lines show energies of S and L types, respectively (see text).

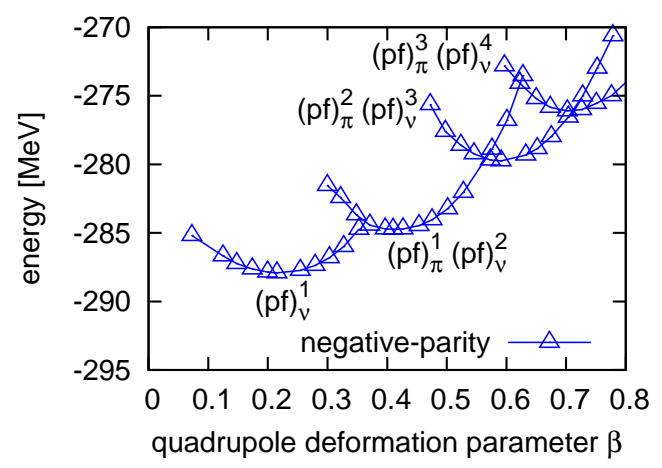

Figure 2: Energy curves as functions of quadrupole deformation parameter $\beta$ obtained by energy variational calculation with parity projection for negative-parity. $(p f)_{\tau}^{n}(\tau=\pi$ or $v)$ shows particle-hole configurations.

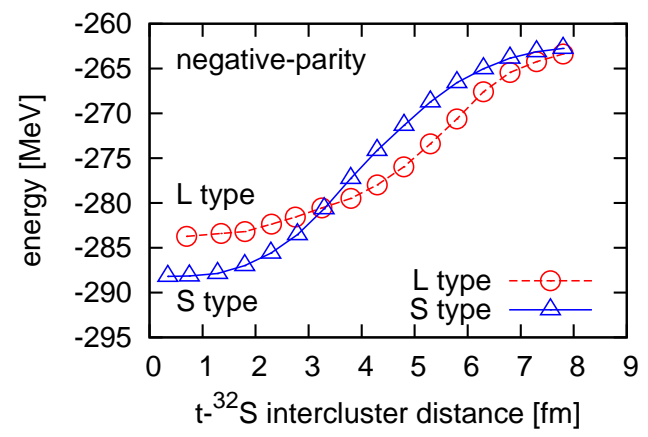

Figure 4: Energies of $t{ }^{-32} \mathrm{~S}$ cluster structure as functions of intercluster distance for negativeparity states. Solid and dotted lines show energies of S and L types, respectively (see text).

$(p f)_{\pi}^{2}(p f)_{v}^{2}$ configurations appear, and their local minima are at $\beta \simeq 0.3$ and 0.5 , respectively. In negative-parity states (Fig. I), four configurations are obtained on the $\beta$-energy surface, which are $(p f)_{\nu}^{1},(p f)_{\pi}^{1}(p f)_{\pi}^{2},(p f)_{\pi}^{2}(p f)_{\pi}^{3}$, and $(p f)_{\pi}^{3}(p f)_{\pi}^{4}$ configurations. The $(p f)_{\nu}^{1},(p f)_{\pi}^{1}(p f)_{\pi}^{2}$, $(p f)_{\pi}^{2}(p f)_{\pi}^{3}$, and $(p f)_{\pi}^{3}(p f)_{\pi}^{4}$ states has local minima at $\beta \simeq 0.2,0.4,0.6$, and 0.7, respectively.

Figure [ $]$ shows energies of negative-parity $\alpha{ }^{3}{ }^{31} \mathrm{P}$ cluster structure as functions of intercluster distance $d_{\alpha^{-31} \mathrm{P}}$ obtained by energy variational calculations with a constraint on $d_{\alpha^{-31} \mathrm{P}}$. By the energy variational calculations, two types of structures are obtained labeled $\mathrm{S}$ and $\mathrm{L}$ types. $\mathrm{A}^{31} \mathrm{P}$ cluster is deformed, and an $\alpha$ cluster is located on the short and long axes for $\mathrm{S}$ and $\mathrm{L}$ types, respectively. In small distance region, energy of S type configuration is lower than that of L type configuration, and the order is exchanged at $d_{\alpha-31 \mathrm{P}} \simeq 4 \mathrm{fm}$. Two configurations have maximum energy at $d_{\alpha-31} \sim 7-8 \mathrm{fm}$, and their energies are similar. The $\mathrm{S}$ and $\mathrm{L}$ type configuration have $1 \hbar \omega$ 


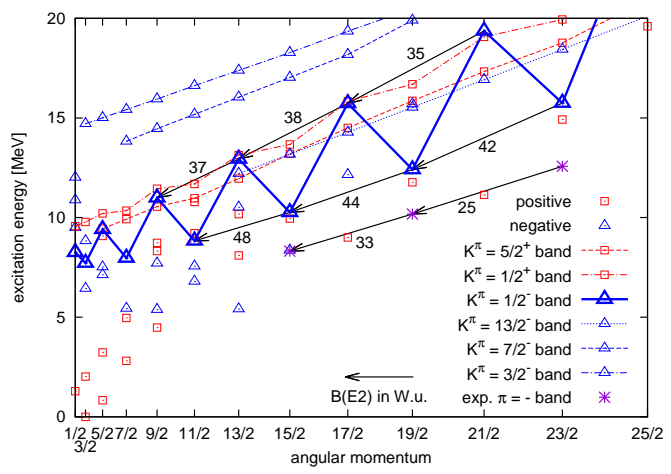

Figure 5: Level scheme and of ${ }^{35} \mathrm{Cl}$. Band members are connected with lines. In-band $B(\mathrm{E} 2)$ values of some bands are written (see text).

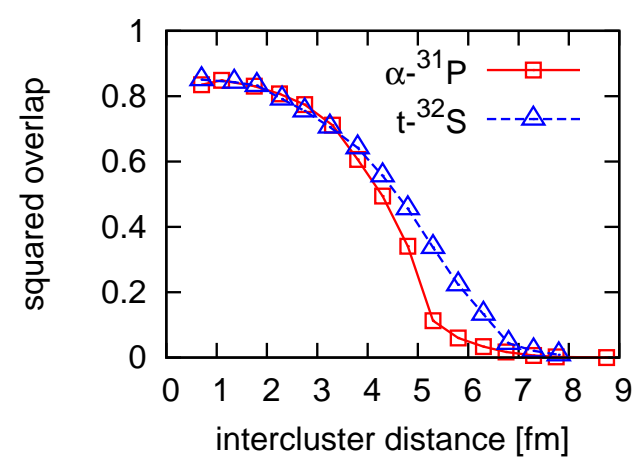

Figure 6: Cluster structure components of the $K=\frac{1}{2}^{-}$band. Solid and dashed lines are for $\alpha$ ${ }^{31} \mathrm{P}$ and $t{ }^{32} \mathrm{~S}$ cluster structure components.

and $3 \hbar \omega$ excited configurations, respectively, in small distance region.

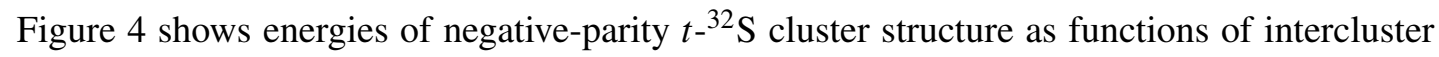
distance $d_{t^{-32} \mathrm{~S}}$ obtained by energy variational calculations with a constraint on $d_{t^{-32} \mathrm{~S}}$. By the energy variational calculations, two types of structures are obtained labeled $S$ and $L$ types. ${ }^{32} S$ cluster is deformed, and an $t$ cluster is located on the short and long axes for $\mathrm{S}$ and $\mathrm{L}$ types, respectively. In small distance region, energy of S type configuration is lower than that of $\mathrm{L}$ type configuration, and the order is exchanged at $d_{\alpha^{-3}{ }^{31} \mathrm{P}} \sim 4 \mathrm{fm}$. All configurations have similar energy in large $d_{t^{-32}} \mathrm{~S}$ region. The $S$ and $L$ type configuration have $1 \hbar \omega$ and $3 \hbar \omega$ excited configurations, respectively, in small distance region.

Figure $\left[\right.$ shows level scheme of ${ }^{35} \mathrm{Cl}$. Various rotational bands are obtained as well as low-lying states. The experimental negative-parity band labeled $\pi=-$ band is also plotted. $B(\mathrm{E} 2)$ values for the theoretical $K^{\pi}=\frac{1}{2}^{-}$band, which is the yrast deformed band, and experimental $\pi=-$ bands are written with arrows. Wave functions around local minima of $\beta$-energy curves become dominant components of rotational bands and other low-lying states. In-band $B(\mathrm{E} 2)$ values of the $K=\frac{1}{2}^{-}$ band are $40 \mathrm{~W}$.u., which is similar to those of the experimental $\pi=-$ band.

The members of $K=\frac{1}{2}^{-}$bands contain large amounts of $\alpha-{ }^{31} \mathrm{P}$ and $t-{ }^{32} \mathrm{~S}$ cluster structure components. The contained components are $\mathrm{L}$ type for both cluster structures. Figure 6 shows $\alpha-{ }^{31} \mathrm{P}$ and $t{ }^{32} \mathrm{~S}$ cluster structure components of $J^{\pi}=\frac{1}{2}^{-}$state in the $K=\frac{1}{2}^{-}$band. The $\alpha{ }^{31} \mathrm{P}$ and $t{ }^{32} \mathrm{~S}$ cluster structure components are similar for small to large intercluster distance. In small distance region, overlaps are more than 0.8 , which means that those cluster structures are dominant components as well as wave functions on the $\beta$-energy surface. With increasing intercluster distance, the overlaps are decreasing, but the overlaps are long tail distribution. Overlaps are still nonneglegible in surface region, $d_{\alpha^{-3} \mathrm{P}, t t^{-32} \mathrm{~S}} \gtrsim 4 \mathrm{fm}$.

\section{Discussions}

The experimental yrast $J^{\pi}=\frac{15}{2}^{-}, \frac{19}{2}^{-}$, and $\frac{23}{2}^{-}$states correspond to members of the theoretical $K^{\pi}=\frac{1}{2}^{-}$band. Excitation energy as functions of $J(J+1)$ is almost straight, and strong E2 
transitions are observed, $B\left(\mathrm{E} 2 ; \frac{19}{2}^{-} \rightarrow \frac{15}{2}^{-}\right)=33$ W.u. and $B\left(\mathrm{E} 2 ; \frac{23}{2}^{-} \rightarrow \frac{19}{2}^{-}\right)=25$ W.u., which shows they are members of deformed band[山]. In theoretical $K^{\pi}=\frac{1}{2}^{-}$band, excitation energy as functions of $J(J+1)$ is also almost straight, and slope the band is similar to that of the experimental $J^{\pi}=\frac{15}{2}^{-}, \frac{19}{2}^{-}$, and $\frac{23}{2}^{-}$states, which shows moment-of-inertia of the experimental and theoretical bands are similar. Furthermore, the experimental and theoretical E2 transition strengths are similar. Dominant components of the $K^{\pi}=\frac{1}{2}^{-}$band have $3 \hbar \omega$ excited configurations, which is consistent with shell-model calculation[四].

Members of the $K^{\pi}=\frac{1}{2}^{-}$band contain large amounts of $\alpha{ }^{-31} \mathrm{P}$ cluster structure components (Fig. (6), which is consistent with a prediction in Ref. [四] for the $K^{\pi}=\frac{1}{2}^{-}$band. The band members also contain similar amounts of $t^{-32} \mathrm{~S}$ cluster structure components as $\alpha-{ }^{31} \mathrm{P}$ cluster structure components though threshold energy of $t+{ }^{32} \mathrm{~S}(17.94 \mathrm{MeV})$ are much higher that that of $\alpha+{ }^{31} \mathrm{P}$ (6.99 MeV). Particle-hole configurations are important for coupling with deformed states. L type $\alpha-{ }^{31} \mathrm{P}$ and $t{ }^{32} \mathrm{~S}$ cluster structures have $3 \hbar \omega$ excited configurations in small distant region, which is same particle-hole configurations as dominant components of the $K^{\pi}=\frac{1}{2}^{-}$band.

\section{Conclusions}

Structures of excited states in ${ }^{35} \mathrm{Cl}$ are investigated by using the AMD and the GCM. The obtained $K^{\pi}=\frac{1}{2}^{-}$band corresponds to the experimental yrast band. $\alpha{ }^{-31} \mathrm{P}$ and $t-{ }^{32} \mathrm{~S}$ cluster structures couple to the $K^{\pi}=\frac{1}{2}^{-}$band, which is explained by particle-hole configurations of their cluster structures in small distant region.

\section{Acknowledgments}

The author thanks to Dr. Kimura in Hokkaido University for fruitful discussions. This work was supported by JSPS KAKENHI Grant Number 25800124. Numerical calculations were performed using COMA at the Center for Computational Sciences, University of Tsukuba.

\section{References}

[1] A. Bisoi, M. S. Sarkar, S. Sarkar, S. Ray, M. R. Basu, D. Kanjilal et al., Superdeformation and $\alpha$-cluster structure in ${ }^{35} \mathrm{Cl}$, Phys. Rev. C 88(2013)034303.

[2] Y. Kanada-En'yo and H. Horiuchi, Clustering in yrast states of ${ }^{20}$ Ne studied with antisymmetrized molecular dynamics, Prog. Theor. Phys. 93(1995) 115.

[3] M. Kimura, Deformed-basis antisymmetrized molecular dynamics and its application to ${ }^{20}$ Ne, Phys. Rev. C 69 (2004)044319.

[4] Y. Taniguchi, M. Kimura and H. Horiuchi, New constraint of clustering for amd and its application to the study of the $2 \alpha-{ }^{12}$ C structure of ${ }^{20}$ Ne, Prog. Theor. Phys. $112(2004) 475$. 